\title{
Estudio de las representaciones sociales de los docentes sobre el cambio climático antropogénico
}

\section{Study of the social representations of the teachers on anthropogenic climate change}

\section{Estudo das representações sociais dos professores sobre mudanças climáticas antropogênicas}

\author{
Adriana Patricia Gallego-Torres ${ }^{1}$ \\ Jhonn Edgar Castro-Montaña ${ }^{2}$
}

Recibido: enero 2020

Aceptado: abril 2020

Para citar este artículo: Gallego-Torres, A.P., Castro-Montaña, J.E. (2020). Estudio de las representaciones sociales de los docentes sobre el cambio climático antropogénico. Revista Científica, 38(2), 229-242. https://doi. org/10.14483/23448350.16190

\section{Resumen}

Este trabajo realizado con los docentes de la Facultad de Ciencias y Educación de la Universidad Distrital Francisco José de Caldas. Tiene como objeto caracterizar las representaciones sociales que los docentes en formación han elaborado sobre el cambio climático antropogénico. Para avanzar en el problema y los objetivos se acudió a plantear una metodología acorde al tipo de estudio social, con un enfoque cualitativo de corte interpretativo, lo que permitió identificar las representaciones sociales de los docentes, que configuran una manera de interpretar $y$ de pensar la realidad cotidiana y s sus actitudes e intereses frente al problema del cambio climático y su inferencia en su labor docente.

Palabras clave: cambio climático, representaciones sociales, enseñanza de las ciencias, docencia.

\begin{abstract}
This work carried out with the teachers of the Faculty of Sciences and Education of the District University Francisco José de Caldas, has as an objective to characterize the social representations that the teachers in formation have elaborated on anthropogenic climate change. In order to advance the problem and the objectives, a methodology according to the type of social study was proposed, with a qualitative approach of interpretive cut, which allowed to identify the social representations of the teachers, which configure a way of interpreting and thinking about the everyday reality and their attitudes and interests in the face of the problem of climate change and its inference in their teaching work.
\end{abstract} Keywords: climate change, social representations, science education, teaching.

1. Universidad Distrital Francisco José de Caldas. Bogotá, Colombia. adpgallegot@udistrital.edu.co

2. Fundación Universitaria Los Libertadores. Universidad Distrital Francisco José de Caldas. Bogotá, Colombia. jecastrom@udistrital.edu.co 


\section{Resumo}

Este trabalho realizado com os professores da Faculdade de Ciências e Educação da Universidade do Distrito Francisco José de Caldas, visa caracterizar as representações sociais que os professores em formação elaboraram sobre mudanças climáticas antropogênicas. Para avançar no problema e nos objetivos, propusemos uma metodologia de acordo com o tipo de estudo social, com uma abordagem qualitativa de natureza interpretativa, que permitiu identificar as representações sociais dos professores, que configuram uma maneira de interpretar e pensar sobre o realidade cotidiana e suas atitudes e interesses em relação ao problema das mudanças climáticas e sua inferência no trabalho de ensino..

Palavras-chaves: cambio climático, representações sociais, sentido das ciências, docência.

\section{Introducción}

Estudiar el cambio climático antropogénico desde las representaciones sociales tiene su trasfondo fundamental ya que éstas son entendidas como sistemas de interpretación, que orientan y organizan las conductas y las comunicaciones sociales; intervienen en procesos como la difusión y la asimilación de conocimientos, el desarrollo individual y colectivo, la definición de las identidades personales y sociales, la expresión de los grupos y las transformaciones sociales (Domínguez, 2006).

El cambio climático Antropogénico es un problema social, lo que hace necesario conocer las representaciones sociales que los docentes en formación han elaborado a través de su interacción con la sociedad y con la enseñanza. En este sentido nuestras conjeturas nos llevan a pensar que el conjunto de conocimientos que los docentes en formación inicial y continua han elaborado sobre el cambio climático obedecen a un sistema complejo de representaciones sociales, asumidas como modelos que encierran una serie de patrones que los ciudadanos utilizan a la hora de tomar decisiones, en nuestro caso relacionadas con mitigar el cambio climático. Teniendo en cuenta esto la pregunta central de nuestra investigación fue:
¿Cuáles son las Representaciones Sociales de los Profesores en Formación de la Facultad de Ciencias y Educación de la Universidad Distrital Francisco José de Caldas sobre el cambio climático antropogénico?

Como se ha dejado planteado, la teoría de las representaciones sociales podría estar íntimamente relacionada con el problema y una posible solución del cambio climático, dado que, las representaciones hacen referencia a objetos que, por su misma naturaleza, contienen la integración de las experiencias individuales subjetivas y de los sistemas de interacción social. Estas representaciones sociales no sólo permiten las interacciones entre los sujetos sociales, sino que les definen posiciones complementarias respecto a los objetos representados y les proporcionan la impresión de formar parte de culturas o comunidades determinadas (Wagner y Elejabarrieta, 1994).

Las representaciones que poseen los docentes en formación sobre el cambio climático son adquiridas y compartidas por la sociedad a través de los medios de comunicación por un lado y por otro las transmitidas por la enseñanza habitual. Como plantea Moscovici (1986) "cada vez que aparece una nueva teoría y capta la imaginación, observamos como miles de personas hablan de ella, intentan comprender su significado y en qué las concierne." Recordemos que las preconcepciones son un conjunto de conocimientos cotidianos que al que los ciudadanos acuden cuando necesitan explicar los fenómenos que los rodean.

Por lo tanto, nos atrevemos afirmar que los docentes en formación de ciencias, como sujetos de una realidad sociocultural, Ilevan al aula las representaciones sociales que han elaborado acerca del cambio climático, ya que muchos de los conceptos asociados al mismo, se han filtrado por los diferentes canales de información.

\section{Fundamentos conceptuales}

Para poder estudiar el problema propuesto se hace necesario acudir por un lado al cambio climático 
antropogénico y por otro a las representaciones sociales como se especifica a continuación:

\section{Cambio Climático Antropogénico}

El cambio climático antropogénico es uno de los problemas más graves a los que se enfrenta hoy la humanidad, sin embargo, a pesar de la amplia difusión que ha tenido en los medios de comunicación masiva, muchos aspectos aun no son claros, dificultando la compresión del problema y la proyección de soluciones viables para hacer frente a dicho problema.

Entre las causas por las cuales se le atribuye al hombre el cambio climático se esgrimen las siguientes:

\section{a. Emisión de gases de efecto invernadero. Uno} de los fenómenos que regula la temperatura promedio de la tierra es el efecto invernadero, descrito y explicado teóricamente en principio en 1800 por el físico Svante Arrhenius y de manera más profunda y formal en 1861 por J. Tyndall. El efecto invernadero consiste básicamente en la retención y emisión de energía térmica a la superficie terrestre y al espacio, por gases que constituyen la atmosfera, que ha sido emitida por la superficie terrestre luego de haber sido irradiada por el sol, es decir que gran parte de energía irradiada por la superficie terrestre es devuelta por la acción de los gases de efecto invernadero, que posteriormente vuelve a ser capturada por dichos gases, configurando un proceso casi cíclico que permite mantener una temperatura promedio determinada (Caballero, Lozano, y Ortega, 2007).

b. El uso de combustibles de origen fósil. El uso de combustibles de origen fósil se ha acentuado históricamente a partir de las revoluciones industriales, la necesidad de producir hierro, acero, combustible para los automóviles y los aviones y generar energía eléctrica, durante el siglo XIX y comienzos del siglo XX, generó un crecimiento vertiginoso del uso de recursos de origen fósil como el carbón mineral y el petróleo, y hoy en día prácticamente bajo las mismas demandas se combina con el uso de gas natural también de origen fósil, lo que ha implicado un aumento en las emisiones de $\mathrm{CO}^{2}$ a la atmosfera (Castro y Gallego, 2015).

c. La deforestación. Otra de las fuentes del calentamiento global antropogénico es la deforestación de grandes zonas donde se realiza la fotosíntesis. A principios del siglo XX el área forestal del planeta era aproximadamente de cinco billones de hectáreas hoy en día quedan algo más o menos que 3.9 billones de hectáreas (Brown, 2008), al disminuir la cantidad de plantas vivas en especial lo que corresponde a bosques, selvas y algas marinas y aumentar las zonas áridas y desérticas, se disminuye uno de los sumideros naturales de $\mathrm{CO}^{2}$ ya que en el procesos de fotosíntesis las plantas convierten la energía radiada por el sol en energía química, en dicho proceso se fija una gran cantidad de dióxido de carbono y se produce oxígeno.

d. La urbanización. En la búsqueda del bienestar, los seres humanos se han concentrado en algunas regiones geográficas donde aceleradamente se aumenta la concentración demográfica. Este proceso de asentamiento generalmente se realiza en lugares con características rurales que anteriormente eran ricos en recursos naturales, lo que implica sin duda algunos procesos de deforestación (NG y REN, 2017). El quebranto de los recursos naturales de las zonas periféricas, como es el caso en Bogotá de los asentamientos realizados en los cerros orientales de la ciudad y en los humedales, desencadenando un aumento en los niveles de contaminación del agua, la falta de una infraestructura tratar las aguas residuales, lo que en muchos casos pone en peligro la vida de los ciudadanos.

e. La industria. Desde el ámbito industrial que tiene un lugar en las ciudades, difícilmente se logra una disposición adecuada de los desechos producidos en sus procesos. Todo lo 
anterior, pone en situación de mayor vulnerabilidad ante desastres naturales (Almazán, 2008).

f. El abuso de la energía eléctrica. Las ciudades se han convertido en grandes consumidores de energía eléctrica para poder satisfacer todas sus necesidades a través de un gran número de artefactos que permanentemente como son las estufas, calentadores, bombillas, computadores, televisores, teléfonos móviles, etc. (Li, Yang y Long, 2018).

g. El trasporte. Un informe realizado por el Instituto Goddard de Estudios Espaciales de la NASA identifica el transporte por carretera como el mayor causante del cambio climático. La generación de energía, aun siendo responsable del mayor impacto a nivel global, también produce un gran número de compuestos que aumentan la reflexividad de las nubes y otros efectos que servirían para reducir o paliar los efectos del calentamiento de los que son responsables.

\section{Representaciones Sociales y el Cambio Climático}

Las representaciones sociales entendidas en sentido genérico como los discursos científicos, filosóficos, políticos, religiosos, etc. conforman el conocimiento cotidiano, que incluye creencias y actitudes socialmente compartidas que les sirven para explicar, clasificar, predecir y, en último término, guían la tomar decisiones (Moscovici, 1976, 1986; Moscovici y Hewstone, 1986; Farra, 1986; Jodelet, 1986, Gaffé, 2004; Lacolla, 2005). Basándonos en que una de las causas del cambio climático, es debido a las acciones del hombre y su relación con la naturaleza, lo que nos lleva a pensar que las representaciones sociales, se forman entre la intersección de la enseñanza de las ciencias y los aprendizajes informales que les ofrece la sociedad, y que pueden convertirse en verdaderos obstáculos epistemológicos, pueden ser una de las causas principales del cambio climático antropogénico (Portillo, et al, 2017; Ballesteros y Gallego, 2019).

Una representación social puede considerarse como "la transformación de lo no familiar en familiar". El pensamiento de sentido común, plagado de teorías implícitas y basado fundamentalmente en lo perceptivo, que intenta recepcionar todo el bombardeo de información acerca de los descubrimientos, las nociones y los lenguajes de la ciencia (Lacolla, 2005). Comprender los mecanismos de formación de las representaciones sociales sobre el cambio climático podría contribuir a mejorar las acciones del hombre con su entorno, bajo una óptica más integral y social (Porras y Pérez, 2018, Gallego, 2018).

Bernardino et al. (2007) comentó que la Teoría de las Representaciones Sociales en el posibilita entender que los diferentes grupos sociales tienen representaciones distintas de un mismo objeto $y$ éste, a su vez, dirige la manera en que se comportan. La formación de las representaciones de un objeto se produce constantemente en la interacción entre los individuos sociales, un proceso Ilamado anclaje y objetivación. Para Sá (1995), en el anclaje, los significados se asignan al objeto de vincular a ella las concepciones y valores existentes, por lo que el desconocido se asocia con una categoría conocida. La objetivación de los conocimientos sobre el objeto se concreta, por lo que el concepto abstracto se convierte en el concreto (Niaz, 2016).

Las representaciones sociales sobre el cambio climático antropogénico pueden ser estudiadas desde tres campos de investigación: Las concepciones sobre el CC, las actitudes y el campo de la representación (Díaz Clemente, 1992; Wagner y Elejabarrieta, 1994; Pereira de Sá, 1998; Mora, 2002; López Alonso y Stefani, 2005, Castro y GaIlego, 2015).

El primero, concierne a la perspectiva inicial de las concepciones sobre el CC. Es decir, las modalidades que el conocimiento cotidiano asume en las sociedades contemporáneas. Como resultado de la secularización creciente de vastos sectores 
sociales, la ciencia, el conocimiento científico, se populariza, se hace parte de la vida cotidiana. (Wagner y Elejabarrieta, 1994). Que no es otra cosa que las representaciones sociales, no solo de la ciencia, sino también de la tecnología y que se van trasformando en la "ciencia popular" que incide sobre la manera de ver el mundo y de actuar de todos quienes pertenecen a una determinada comunidad (Gutiérrez, 2016).

El segundo campo de investigación se refiere al estudio de la de la influencia social y cultural. Se trata de aquellos fenómenos e interacciones cuyas representaciones tienen un arraigo y han estado presentes en los seres humanos durante mucho tiempo. Estas representaciones sociales no sólo permiten las interacciones entre los sujetos sociales, sino que les definen posiciones complementarias respecto a los objetos representados y les proporcionan la impresión de formar parte de culturas o comunidades determinadas (Wagner y Elejabarrieta, 1994). Entre sus características merece destacarse que son construcciones mentales que actúan como motores del pensamiento, que funcionan y perduran con independencia de tales o cuales individuos concretos y generan conductas relacionadas con ellas (Lacolla, 2005).

Un tercer campo de investigación hace referencia a la actitud de los ciudadanos frente al problema del cambio climático. Acudiendo a Banchs, este campo separa los desarrollos teóricos de las representaciones sociales. Concierne a los diferentes presupuestos ontológicos y epistemológicos y axiológicos que asumen la vertiente procesual y la vertiente estructural (Banchs, 2000). Lo que conlleva a reflexionar en torno a la actitud hacia el CC, Las actuaciones individuales, de grupo o sociales en relación con la generación, aplicación o transformación del proceso de construcción del conocimiento racional, empírico y objetivo.

Desde esta perspectiva, podemos acudir a las representaciones para el planteamiento de propuestas que conlleven a las nuevas generaciones a adquirir comportamientos, valores e interés más acordes con la necesidad de mitigar el cambio climático.
Establecer las actitudes, los valores o los intereses que se relacionan e interaccionan con el fenómeno del cambio climático es uno de los problemas actuales por resolver en la enseñanza de las ciencias y no es otro que establecer planteamientos innovadores desde la didáctica de las ciencias que integren la historia, la filosofía, la sociología, etc. Las cuales, muy seguramente determinaran nuevos matices en la lucha global por la supervivencia de la especie humana (Ballesteros y Gallego, 2019).

Si partimos del hecho de que las representaciones sociales tienen un componente conceptual (el modelo del cambio climático) un componente actitudinal (las actitudes y los valores) como base la representación social podría explicarse la actitud pasiva de algunos colectivos hacia el cambio climático como el resultado de una representación social es necesario que se ha ido construyendo acerca del problema.

\section{Metodología}

En concordancia con los objetivos de la investigación el tipo de estudio adoptado es estadístico-descriptivo de corte cuantitativo, que mide cómo es y cómo se manifiesta el fenómeno de las representaciones sociales.

\section{Enfoque Metodológico}

En tanto el objeto de este estudio es un fenómeno social, el enfoque es mixto cualitativo de corte interpretativo, en ese sentido, Vasilachis sostiene al respecto de la investigación cualitativa "[...] permite al especialista satisfacer todos los requisitos básicos de la ciencia empírica: enfrentarse a un mundo susceptible de observación y análisis, suscitar problemas con respecto al mismo, reunir los datos necesarios a través de un examen detenido y disciplinado, descubrir relaciones entre las respectivas categorías de los datos, formular proposiciones respecto de esas relaciones, incorporarlas a un sistema teórico, y verificar problemas, datos, 
relaciones, proposiciones y teorías por medio de un nuevo examen del mundo empírico." (Vasiliachis, 1992, Hurtado, 2014). Desde esta perspectiva La teoría fundamentada es uno de los enfoques de la metodología cualitativa que permite crear propuestas teóricas basándose exclusivamente en los datos lo que permite la categorización, la recolección de datos y el análisis de las representaciones de los docentes sobre el cambio climático (Glaser y Strauss, 1967)

\section{Variables y unidades de análisis para el estudio de las representaciones sociales de los docentes sobre el cambio climático}

Para la recolección de la información se procedió a la elaboración de un cuestionario que nos permitiera conocer y analizar las diferentes representaciones sociales en los docentes en formación inicial de las licenciaturas de física, química y biología de la Facultad de Educación de la Universidad Distrital Francisco José de Caldas conformado por 28 preguntas. Para ello se seleccionaron de 3 grupos de docentes quienes ya habían realizado al menos una práctica docente.

A partir de la fundamentación teórica de la investigación, las categorías que emergieron se ubicaron en torno a tres categorías, que quedaron identificadas y definidas tal y como se pueden observar en la tabla 1.

\section{Resultados}

Para poder realizar el análisis estadístico se seleccionaron un conjunto de setenta estudiantes de diferentes proyectos curriculares de la facultad de ciencias y educación. A continuación, se realiza un análisis interpretativo, de algunas estadísticas calculadas sobre las respuestas dadas en la encuesta (28 ítems), elaborada por el grupo de investigación IREC donde cada ítem presentaba 5 posibles respuestas: muy en desacuerdo (1), en desacuerdo (2), indiferente (3), de acuerdo (4) y muy de acuerdo (5).

Población (N): Docentes en formación. Universidad Distrital Fráncico José de Caldas.

Muestra (n): 70 estudiantes seleccionados aleatoriamente.

Objetivo: Identificar concepciones sobre cambio climático adquiridas en el proceso de formación docente.

Forma de recolección de la información: Encuesta.

\section{Análisis estadístico grupal por dimensiones}

En el siguiente apartado, se presentan los resultados del análisis de las preguntas realizadas en las encuestas, el propósito principal consiste en analizar desde una perspectiva que agrupe las preguntas teniendo como criterio las tres dimensiones

Tabla 1. Categorías identificadas

\begin{tabular}{llll}
\hline \multicolumn{1}{c}{ Categoría } & \multicolumn{1}{c}{ Definición Operacional } & Dimensión & Indicador \\
\hline $\begin{array}{l}\text { Representaciones del } \\
\begin{array}{l}\text { Cambio climático } \\
\text { antropogénico }\end{array}\end{array}$ & $\begin{array}{l}\text { ¿Qué es el cambio climático } \\
\text { antropogénico? }\end{array}$ & $\begin{array}{l}\text { Aspectos conceptuales } \\
\text { del CCA }\end{array}$ & $\begin{array}{l}\text { Identifica y reconoce las } \\
\text { problemáticas asociadas a } \\
\text { los parámetros del CCA }\end{array}$ \\
\hline $\begin{array}{l}\text { Representaciones sobre } \\
\text { aspectos Históricos, } \\
\text { sociales y culturales. }\end{array}$ & $\begin{array}{l}\text { Relaciones, históricas, } \\
\text { culturales y sociales del cambio } \\
\text { climático antropogénico que } \\
\text { conforman las actitudes. }\end{array}$ & $\begin{array}{l}\text { Fundamentos } \\
\text { actitudinales, } \\
\text { comportamentales frente } \\
\text { al CC }\end{array}$ & $\begin{array}{l}\text { Identifica y reconoce, los } \\
\text { temas relacionados las } \\
\text { causas y los efectos del CCA }\end{array}$ \\
$\begin{array}{l}\text { Representaciones sobre } \\
\begin{array}{l}\text { Cambio climático la } \\
\text { formación docente. }\end{array}\end{array}$ & $\begin{array}{l}\text { Representaciones sobre el CC } \\
\text { conformadas por las actitudes, } \\
\text { valores y creencias de los docentes } \\
\text { en formación frente al CCA }\end{array}$ & $\begin{array}{l}\text { Aspectos educativos, } \\
\text { Axiológicos y } \\
\text { actitudinales que } \\
\text { conforman la RS }\end{array}$ & $\begin{array}{l}\text { Determinar las RS del CCA } \\
\text { en la formación docente que } \\
\text { reconocen los profesores }\end{array}$ \\
\hline
\end{tabular}

Fuente: Los autores 
construidas a partir de las categorías teóricas. Primero se calculó para cada una de las preguntas el promedio a partir de las valoraciones entregadas por los 69 estudiantes. Luego se agruparon todas aquellas preguntas que están enfocadas a describir la dimensión, y a partir de la comparación de las medianas utilizando como método estadístico la prueba de Kruskall y Wallis se desarrolló un análisis de varianza desde una perspectiva no paramétrica.

\section{Representaciones conceptuales sobre el CCA}

La primera categoría hace referencia a las representaciones sociales conceptuales que los docentes en formación han elaborado sobre el cambio climático y que hemos denominado aspectos conceptuales sobre el CCA, en esta dimensión se ubicaron un total de nueve preguntas, a continuación, se presenta una tabla 2 que reúne las preguntas y su rango promedio.

El valor de la estadística de prueba para este conjunto de preguntas es de 316,752. El p-valor es menor del 0,05, lo que indica que existe diferencia significativa entre las puntuaciones de las medianas de las valoraciones entregadas por los estudiantes agrupados en este conjunto de preguntas.
La menor valoración se encontró a la pregunta No me interesan las consecuencias del cambio climático con un rango promedio de 113,8, y a La naturaleza tiene una capacidad inagotable y se puede recuperar fácilmente de los daños provocados por las acciones humanas, con 132,5 lo que indica que los estudiantes se encuentran preocupados por los problemas relacionados con el cambio climático. Lo cual concuerda con los valores entregados a las preguntas que indagaban sobre las consecuencias que se podrían derivar del cambio climático entre ellas; Las acciones que afectan actualmente el equilibrio del planeta tendrán drásticas consecuencias en el futuro con un rango promedio de 469,7 y La mayoría de las personas deben cambiar su manera de actuar con relación al cambio climático con 458,9.

\section{Aspectos actitudinales sobre el CCA}

La segunda agrupación corresponde a la dimensión: los aspectos históricos, sociales y culturales del problema del cambio climático antropogénico. Con el propósito de analizar en profundidad esta dimensión, se realizó una interpretación de aquellas preguntas que estaban enfocadas al aspecto mencionado. Las preguntas seleccionadas se presentan en la tabla 3.

Tabla 2. Preguntas y su rango promedio.

\begin{tabular}{|c|c|c|}
\hline Pregunta & $\begin{array}{l}\text { Numero de } \\
\text { datos }\end{array}$ & $\begin{array}{c}\text { Rango } \\
\text { promedio }\end{array}$ \\
\hline $\begin{array}{l}\text { El conocimiento que he construido sobre el cambio climático es el necesario para comprender } \\
\text { sus causas, consecuencias y participar en el desarrollo de alternativas que permitan hacerle frente. }\end{array}$ & 69 & 329,978 \\
\hline No me interesan las consecuencias del cambio climático & 69 & 113,884 \\
\hline Colombia es un país que debe considerarse como un proveedor de riquezas naturales infinitas. & 69 & 318,065 \\
\hline El bienestar de las personas depende de la capacidad de consumo de bienes materiales & 69 & 206,145 \\
\hline La mayoría de las personas deben cambiar su manera de actuar con relación al cambio climático & 69 & 458,993 \\
\hline $\begin{array}{l}\text { La naturaleza tiene una capacidad inagotable y se puede recuperar fácilmente de los daños } \\
\text { provocados por las acciones humanas }\end{array}$ & 68 & 132,529 \\
\hline Incluyo acciones en mi vida que permiten minimizar los efectos del cambio climático. & 69 & 386,486 \\
\hline $\begin{array}{l}\text { Las acciones que afectan actualmente el equilibrio del planeta tendrán drásticas consecuencias } \\
\text { en el futuro }\end{array}$ & 69 & 469,797 \\
\hline $\begin{array}{l}\text { Intento reducir el consumo de los diferentes bienes que ofrece la sociedad actual, con el fin de } \\
\text { disminuir mi huella ecológica sobre el planeta }\end{array}$ & 69 & 372,456 \\
\hline
\end{tabular}

Fuente: Los autores 
Tabla 3. Preguntas seleccionadas

\begin{tabular}{|c|c|c|c|c|}
\hline Pregunta & & Promedio & $\begin{array}{l}\text { Desviación } \\
\text { Estándar }\end{array}$ & Coef. of variación \\
\hline No me interesan las consecuencias del cambio climático & 69 & 1,27 & 0,70 & $55 \%$ \\
\hline $\begin{array}{l}\text { Las personas exageran al decir que son graves las consecuencias de } \\
\text { contaminar la atmosfera cuando se hace uso del automóvil como medio } \\
\text { de transporte. }\end{array}$ & 69 & 1,78 & 0,98 & $55 \%$ \\
\hline $\begin{array}{l}\text { El consumo excesivo de los recursos ha venido agravando la problemática } \\
\text { ambiental actual. }\end{array}$ & 69 & 4,40 & 1,01 & $23 \%$ \\
\hline $\begin{array}{l}\text { El manejo adecuado de las basuras es responsabilidad exclusiva de las } \\
\text { empresas de aseo. }\end{array}$ & 69 & 1,42 & 0,77 & $54 \%$ \\
\hline $\begin{array}{l}\text { Colombia es un país que debe considerarse como un proveedor de } \\
\text { riquezas naturales infinitas. }\end{array}$ & 69 & 3,04 & 1,57 & $51 \%$ \\
\hline $\begin{array}{l}\text { Los procesos de autorregulación que mantienen el equilibrio de la } \\
\text { naturaleza son lo suficientemente flexibles para asumir los impactos de la } \\
\text { producción de las multinacionales. }\end{array}$ & 68 & 1,82 & 1,05 & $57 \%$ \\
\hline $\begin{array}{l}\text { El bienestar de las personas depende de la capacidad de consumo de } \\
\text { bienes materiales. }\end{array}$ & 69 & 2,02 & 1,11 & $54 \%$ \\
\hline $\begin{array}{l}\text { La mayoría de las personas deben cambiar su manera de actuar con } \\
\text { relación al cambio climático. }\end{array}$ & 69 & 4,27 & 0,90 & $21 \%$ \\
\hline $\begin{array}{l}\text { La naturaleza tiene una capacidad inagotable y se puede recuperar } \\
\text { fácilmente de los daños provocados por las acciones humanas. }\end{array}$ & 68 & 1,41 & 0,88 & $62 \%$ \\
\hline $\begin{array}{l}\text { Los ciudadanos del común no son responsables del aumento de la } \\
\text { temperatura del planeta. }\end{array}$ & 68 & 2,02 & 1,34 & $66 \%$ \\
\hline $\begin{array}{l}\text { Hacer frente al cambio climático es una responsabilidad exclusiva de los } \\
\text { gobiernos y no de los ciudadanos. }\end{array}$ & 69 & 1,53 & 0,93 & $60 \%$ \\
\hline $\begin{array}{l}\text { Incluyo acciones en mi vida que permiten minimizar los efectos del } \\
\text { cambio climático. }\end{array}$ & 69 & 3,75 & 0,75 & $20 \%$ \\
\hline $\begin{array}{l}\text { Las acciones que afectan actualmente el equilibrio del planeta tendrán } \\
\text { drásticas consecuencias en el futuro. }\end{array}$ & 69 & 4,31 & 1,13 & $26 \%$ \\
\hline $\begin{array}{l}\text { Es necesario que se revisen los procesos de fabricación de insumos y } \\
\text { comprobar que cuenten con los estándares de sostenibilidad. }\end{array}$ & 68 & 4,27 & 0,86 & $20 \%$ \\
\hline $\begin{array}{l}\text { Intento reducir el consumo de los diferentes bienes que ofrece la sociedad } \\
\text { actual, con el fin de disminuir mi huella ecológica sobre el planeta. }\end{array}$ & 68 & 3,61 & 0,84 & $23 \%$ \\
\hline $\begin{array}{l}\text { Reconozco el impacto que sobre el sistema planetario tiene la producción } \\
\text { de los artefactos tecnológicos que utilizo a diario. }\end{array}$ & 69 & 3,75 & 0,99 & $26 \%$ \\
\hline
\end{tabular}

Fuente: Los autores.

Como resultado del cálculo de los promedios y su correspondiente desviación estándar para cada una de las preguntas se encontró, que en general, los encuestados no son indiferentes a los problemas causados por el cambio climáti$\mathrm{CO}$, ya que otorgaron valoraciones muy bajas a la pregunta seis No me interesan las consecuencias del cambio climático con un promedio de 1,27 y una desviación estándar bastante baja 0,70. Esta idea está fuertemente asociada a la intención observada en relación que es necesario el cambio de actitudes por parte de las personas y sus costumbres, de hecho la pregunta la mayoría de las personas deben cambiar su manera de actuar con relación al cambio climático, fue calificada con valores altos 4,27 y desviación de 0,9. También es posible inferir en el mismo sentido que los encuestados le otorgan una gran cantidad de culpa al calentamiento global a las costumbres de los ciudadanos, por tanto, dieron valoraciones muy bajas a las preguntas, los ciudadanos del común no son responsables del aumento de la temperatura con media aritmética de 2,02 y desviación 1,34. 
Pero además advierten que la responsabilidad debe ser compartida con los gobiernos cuando asignan calificaciones muy bajas en la pregunta hacer frente al cambio climático es una responsabilidad exclusiva de los gobiernos y no de los ciudadanos con promedio de 1.53 y desviación de 0.93, lo cual indica que hay una amplia aceptación de la influencia de las personas dentro de los problemas relacionados con el calentamiento global. De la misma manera el promedio para la pregunta nueve Las personas exageran al decir que son graves las consecuencias de contaminar la atmosfera cuando se hace uso del automóvil como medio de transporte, fue de 1.78 con desviación estándar de 1,78, indica una fuerte preocupación por el uso de combustibles fósiles.

Con relación al problema del consumo y su correspondiente efecto sobre la cantidad de desperdicios, se puede observar que existe un alto grado de intranquilidad, ya que los valores promedio para la pregunta El manejo adecuado de las basuras es responsabilidad exclusiva de las empresas de aseo fue de 1,42 y desviación estándar de 0,77, idea que está en correspondencia con las altas valoraciones entregadas en la pregunta El consumo excesivo de los recursos naturales ha venido agravando la problemática ambiental, que fueron de 4,4 y desviación estándar de 1,01.

De todas maneras, los encuestados no se encuentran muy convencidos con relación a la transformación o cambio de sus hábitos, actuaciones o conductas que permitan una menor influencia en el cambio climático actualmente, ya que sus valoraciones para las preguntas que indagaban sobre este tópico fueron intermedias. En la pregunta incluyo acciones en mi vida que permiten minimizar los efectos del cambio climático la media aritmética fue de 3,75 y desviación estándar de 0,75, de la misma manera, cuando se indaga sobre si están dispuestos a realizar una reducción en el consumo, elemento esencial para tratar de detener los efectos del calentamiento global se encontró una situación similar, porque a la pregunta intento reducir el consumo de los diferentes bienes que ofrece la sociedad actual, con el fin de disminuir mi huella ecológica sobre el planeta la media también fue muy parecida 3,61 y desviación de 0,84. Este escenario contrasta de todas maneras con el hecho que los encuestados son conscientes que los efectos se verán en un tiempo posterior, ya que a la pregunta las acciones que afectan actualmente el equilibrio del planeta tendrán drásticas consecuencias en el futuro, asignaron valoraciones altas 4,31 y la desviación fue de 1,13 . Tal vez la alternativa que proponen que puede ser efectuada rápidamente está relacionada con la idea de desarrollar procesos donde la producción sea más limpia, en efecto a la pregunta Es necesario que se revisen los procesos de fabricación de insumos y comprobar que cuenten con los estándares de sostenibilidad, la cual fue valorada con 4,27 con desviación de 0,86.

\section{Representaciones sociales del cambio climático como eje transversal}

Los resultados se presentan en la siguiente tabla, responden a la tercera dimensión, que hace referencia a la transversalidad del modelo del cambio climático en la Facultad de Ciencias y Educación y la hemos denominado: El cambio climático como eje transversal.

La prueba Kruskall Wallis prueba la hipótesis nula que todas las medianas de las valoraciones por pregunta son iguales y se basa en la asignación de rangos para cada una de las preguntas, el valor de la estadística de prueba es entonces iguala a 312,357, que alcanza un p-valor menor al 0.05, lo que nos indica que existe diferencia significativa entre las valoraciones entregadas por pregunta.

A partir de los contrastes múltiples se encontró que los docentes en formación inicial le asignan una importante valoración al hecho que la escuela debe asumir la responsabilidad de hacer frente al cambio climático, de la misma manera esperan que la Facultad de ciencias y educación debería ofrecer espacios académicos relacionados con el cambio climático, pero también le entregan una gran responsabilidad a los profesores en el trabajo, 
Tabla 4. Tranversalidad del modelo climático en la Facultad de Ciencias y Educación.

\begin{tabular}{lcc}
\hline \multicolumn{1}{c}{ Pregunta } & $\begin{array}{c}\text { Numero de } \\
\text { datos }\end{array}$ & Rango promedio \\
\hline $\begin{array}{l}\text { Siento que en el proyecto curricular me ofrecen suficiente formación sobre el problema } \\
\text { del cambio climático. }\end{array}$ & 69 & 168,094 \\
\hline $\begin{array}{l}\text { Las publicaciones científicas, artículos e información en los medios de comunicación } \\
\text { sobre el cambio climático son escasas }\end{array}$ & 69 & 272,732 \\
\hline $\begin{array}{l}\text { Los problemas concernientes con el cambio climático no son importantes en mi vida } \\
\text { académica }\end{array}$ & 67 & 98,6493 \\
\hline $\begin{array}{l}\text { Los problemas relacionados con el cambio climático son requisito en mi formación } \\
\text { académica }\end{array}$ & 69 & 358,391 \\
\hline $\begin{array}{l}\text { Pienso que la Facultad de Ciencias y Educación debería ofrecer espacios académicos } \\
\text { relacionados con el cambio climático. }\end{array}$ & 69 & 445,754 \\
\hline $\begin{array}{l}\text { La Facultad de Ciencias y Educación induce al estudio de los problemas del cambio } \\
\text { climático. }\end{array}$ & 68 & 198,816 \\
\hline $\begin{array}{l}\text { Pienso que la escuela también debería asumir la responsabilidad de hacer frente al } \\
\text { cambio climático }\end{array}$ & 69 & 450,565 \\
\hline $\begin{array}{l}\text { Los profesores no asumen de manera adecuada el cambio climático en las clases } \\
\text { porque le hace falta formación al respecto }\end{array}$ & 69 & 344,312 \\
\hline $\begin{array}{l}\text { Los profesores deben asumir la responsabilidad de trabajar el cambio climático en las } \\
\text { aulas escolares. }\end{array}$ & 69 & 440,471 \\
\hline
\end{tabular}

Fuente: Los autores.

lo que está fuertemente asociado con la creencia que los docentes no asumen de manera adecuada el cambio climático en sus clases. De la misma manera, están muy desacuerdo con la idea que los problemas concernientes con el cambio climático no son importantes en su vida académica.

Los resultados obtenidos arrojaron en resumen las siguientes representaciones sociales:

RS Conocimiento Cotidiano: Los profesores de la Facultad de Ciencias y Educación, poseen una idea vaga en torno al modelo científico del cambio climático y sus elaboraciones son fruto de la popularización que se hecho del mismo y que en muchos casos es obtenida de los programas televisivos de divulgación y de la red, lo que lleva a determinar que la RS es fruto del conocimiento cotidiano. Los profesores en formación inicial le asignan una importante valoración al hecho que la escuela debe asumir la responsabilidad de hacer frente al cambio climático, lo que está fuertemente asociado con la creencia que los docentes no asumen de manera adecuada el cambio climático en sus clases. Lo que ha Ilevado a contribuir a la trasmisión de teorías implícitas sobre el CC y en el peor de los casos a errores conceptuales que por su condición de docentes de ciencias, serán trasmitidas por ellos a las siguientes generaciones

RS Modelo Científico CC: La formación científica que ellos han obtenido no les haya posibilitado adentrarse en la problemática y, por tanto, son poco rigurosos sus conocimientos alrededor del CC. El modelo científico sobre el cambio climático no se desarrollado teniendo en cuenta los factores socio- culturales, lo que llevaría a que los conocimientos y las representaciones elaboradas sean poco consistentes. El agravante de estas representaciones mayormente cotidianas se hace más grave al ser la educación superior y más aún en la formación docente una de las principales responsables de construir conocimientos científicos rigurosos, pertinentes y de calidad sobre el Cambio Climático. Es preciso formular propuestas didácticas que permitan conocer y reflexionar sobre los procesos de formación en educación en ciencias en torno al CC que permitan desde una perspectiva cultural y representacional la trasmutación de los modelos cotidianos a los modelos de representación científica del CC. 
Entre los docentes participantes en el estudio se pudo notar errores conceptuales entre el cambio climático y otros problemas ambientales relacionados como son: la capa de ozono, la lluvia ácida, la contaminación atmosférica, entre otros. Lo que nos refuerza el hecho de que no han construido un modelo científico sobre el CC.

RS Actitudes Frente al CC: Aunque en muchas ocasiones reconocen que no realizan ninguna acción para mitigar el problema del CC, también reconocen contribuir con el efecto invernadero, ya que desconocen en la mayoría de los casos, que sus actos contribuyen al calentamiento del planeta. En este sentido, sus representaciones frente a la huella ecológica son bastante pobres, hasta Ilegar al punto de no reconocerla como parte del problema y no sentirse plenamente involucrados en tomar conciencia y/o actitudes positivas hacia el problema del cambio climático y el calentamiento global, esto se ve claramente representado en el hecho de que los docentes, no se encuentran muy convencidos en relación a la transformación o cambio de sus hábitos, actuaciones o conductas que permitan una menor influencia en el cambio climático actualmente, ya que sus valoraciones para las preguntas que indagaban sobre este tópico fueron intermedias. Esto se debe principalmente a que los docentes derivan el problema a los gobiernos y a las políticas públicas, la gran mayoría opina que no es responsabilidad de la escuela ni de los ciudadanos asumir el problema del cambio climático, lo que nos lleva asumir, que dentro de sus representaciones sociales sobre el cambio climático no asumen la responsabilidad del ser humano como principal detonador del problema. Por el contrario, son bastante indiferentes a tomar partida frente a la necesidad de frenar las emisiones de gas carbónico y cuidar los recursos naturales, entre otros.

RS cambio climático antropogénico: En Los docentes no se evidencian los conceptos claves para comprender el campo climático antropogénico. Lo que supone que ellos no han elaborado un modelo ciudadano que les permita adoptar medidas para mitigar los graves efectos del cambio climático. Esto se evidencia en que ellos no se hacen responsables, del consumo excesivo de los recursos naturales, la deforestación, la contaminación, las emisiones de $\mathrm{CO}_{2}$, el gasto energético, etc. Todo esto producto de la falta de formación y una postura clara y coherente frente al modelo antropogénico. De este modo, aun aceptando la magnitud del problema, los docentes no están convencidos de la necesidad de contribuir a su solución y de promover entre sus estudiantes y futuros ciudadanos comportamientos y actitudes que permitan a las nuevas generaciones vivir en condiciones decorosas.

En los docentes no se manifiesta un puente entre el modelo científico sobre el CC y la percepción del riesgo a las actividades de la vida diaria con la excepción, a acciones puntuales sustitución de bombillos ahorradores, electrodomésticos bajo consumo y el reciclaje de las basuras, etc.

En resumen, las Representaciones Sociales sobre el cambio climático y el calentamiento global, están permeadas por el conocimiento cotidiano, lo que agrava el problema dadas las implicaciones sociales y culturales de los docentes frente a las nuevas generaciones.

En este sentido, hemos de resaltar la necesidad de abordar el problema del cambio climático y el calentamiento global desde la didáctica de las ciencias experimentales; proceso que debe comenzar con la introducción del modelo científico sobre el cambio climático en los programas de formación inicial y continua de profesores de ciencias, no como una asignatura sino como un eje transversal de los currículos, ya que debe ser visto desde la complejidad ambiental que sugiere una nueva epistemología donde se fundan los valores y se interdisciplinen los saberes, además, se requiere de transposiciones didácticas adecuadas, desde la perspectiva del movimiento CTSA, ya que además de la introducción de los modelos, no podemos dejar de lado, los valores, las actitudes, los imaginarios, los intereses, las concepciones, entre otros. De este modo, el énfasis en fomentar la 
construcción de un modelo científico sobre el CC no necesariamente llevará a los docentes a adoptar comportamientos y actitudes sobre el estilo de vida que contribuye a mitigar el CC y a adquirir una formación científica ciudadana que incremente la responsabilidad individual sobre el problema y sobre la huella que cada uno de ellos deja sobre el planeta y la semilla que siembra en sus estudiantes.

\section{Conclusiones}

El enfoque metodológico, permitió determinar que los docentes en formación de la Facultad de Ciencias y Educación a través de un cuidadoso análisis estadístico de la información, nos permitió establecer las siguientes Representaciones Sociales basadas en las tres categorías emergentes establecidas.

A partir de los análisis realizados, podemos concluir, que lo que los docentes en sus clases trasmiten a sus estudiantes, va más allá de las concepciones sobre el modelo del cambio climático y el calentamiento global, convirtiéndose en Representaciones Sociales del mismo, al tener componentes teóricos y actitudinales. Acudiendo a lo propuesto por Lacolla en 2005 "la transformación de lo no familiar en familiar". El pensamiento de sentido común, plagado de teorías implícitas y basado fundamentalmente en lo perceptivo, que intenta recepcionar todo el bombardeo de información acerca del cambio climático, las nociones y los lenguajes del modelo.

\section{Referencias}

Ballesteros-Ballesteros, V., Gallego-Torres, A.P.(2019). La educación en energías renovables desde las controversias socio-científicas en la educación en ciencias. Revista Científica, 2(35), 192-200. https://doi.org/10.14483/23448350.14869

Ballesteros-Ballesteros, V., Gallego-Torres, A. (2019). Modelo de educación en energías renovables desde el compromiso público y la actitud energética. Revista Facultad
De Ingeniería, 28(52), 27-42. https://doi. org/10.19053/01211129.v28.n52.2019.965

Banchs, M. A. (2000). Aproximaciones procesuales y estructurales al estudio de las representaciones sociales. En: Papers on social representations. 9(3):1-15.

Barona, J. L. (1994). Ciencia e historia. Debates y tendencias en la historiografía de la ciencia. Valencia: Guada.

Caballero, M., lozano, S., Ortega, B. (2007). Efecto invernadero, Calentamiento Global y cambio climático: Una perspectiva desde las ciencias de la tierra. Revista digital universitaria , 8(10), 2-12

Capuano, V. Y., Martín, J. (2007). El calentamiento global del planeta Tierra: un ejemplo de equilibrio dinámico. Revista de Enseñanza de la Física 20(1-2), 91-110.

Castro-Montaña, J. E., Gallego-Torres, A. P. (2015). La educación energética una prioridad para el milenio. Revista Científica, 1(21), 97110. https://doi.org/10.14483/udistrital.jour. $\underline{\text { RC.2015.21.a11 }}$

Chevallard, Y. (1985). La transposition didactique. Du savoir savant au savoir enseigné. Grenoble: La Pensée Sauvage.

Díaz-Clemente, M. (1992). Psicología Social (Métodos y Técnicas de investigación). Madrid- España, Ed. Eudema. pp. 391-400.

Domínguez-Gutiérrez, S. (2006). Las representaciones sociales en los procesos de comunicación de la ciencia, I Congreso Iberoamericano de Ciencia, Tecnología, Sociedad e Innovación CTS+ 1, Ponencia presentada en el Palacio de Minería, México.

Echeverría, J. (1998). Filosofía de la ciencia. Madrid: Akal.

Farra, P. (2009). Breve historia de la ciencia. Madrid: Ariel

Bernardino, C. F., Freire, L. M., Ferreira, A., \& Ruiz, R. (2007). Análise das representações sociais sobre meio ambiente de técnicos e professores das Secretarias de Educação e Meio Ambiente de municípios da Bacia de Campos - RJ. In: 
Encontro Nacional De Pesquisa Em Educação Em Ciências, 6., 2007, Florianópolis. Brasil, Florianópolis. ENPEC,

Ferreira, V.C.P., Santos Júnior, A.F., Azevedo, R.C., Valverde, G. (2005). A representação social do trabalho: uma contribuição para o estudo da motivação. Estação científica, 1, 1-13.

Gallego-Torres, A. P. (2018). Las representaciones sociales, un concepto a tener en cuenta. Revista Científica, 2(32), 128. https://doi. org/10.14483/23448350.13301

Graca, M., Moreira, M. A. y Caballero, C. (2004). Representacoes sobre a Matemática, seu ensino e aprendizagem: um estudo exploratório. Revista Investigacoes em Ensino de Ciencias, 9, 1, 37-93, 2004.

Gutiérrez-Sabogal, L. H. (2016). Problemática de la educación ambiental en las instituciones educativas. Revista Científica, 3(23), 5776. https://doi.org/10.14483/udistrital.jour. RC.2015.23.a5

Hurtado, G. E. (2014). ¿Cuáles son las tendencias en las metodologías de enseñanza de la última década en iberoamérica? Revista Científica, 1(18), 86 - 99. https://doi. org/10.14483/23448350.5564

López-Rivera, Z. C. (2015). La Enseñanza de las Ciencias Naturales desde el enfoque de la Apropiación Social de la Ciencia, la Tecnología y la Innovación ASCTI en la educación básica-media. Revista Científica, 2(22), 75-84. https://doi.org/10.14483/10.14483/udistrital. jour.RC.2015.22.a6

Lacolla, L. (2005). Representaciones sociales: una manera de entender las ideas de nuestros alumnos. Revista Electrónica de la Red de Investigación Educativa 1, (3), 1-17.

Li, J., Yang, I., Long, H. (2018). Climatic impacts on energy consumption: Intensive and extensive margins. Energy Economics, 71(1), 332-343. https://doi.org/10.1016/j.eneco.2018.03.010

López Alonso, A. O., Stefani, D. (2005). Representaciones sociales de la vida: su variación a través del género y la edad de las personas.
Su convergencia y divergencia. Revista Signos Universitarios, 41, 23-118.

Martínez Filomeno, M. S. (2003). La representación social de la profesión docente: creencias respecto de la ciencia y la enseñanza. Buenos Aires: Memorias de las Primeras Jornadas sobre Representaciones Sociales, Ciclo Básico Común, Universidad de Buenos Aires.

Mora, M. (2002). La teoría de las Representaciones Sociales de Serge Moscovici. Athenea Digital. Revista de pensamiento e investigación social, 1(2). https://doi.org/10.5565/rev/athenea.55

Moscovici, S. (1976). "Psicología Social" Tomos I y II. Edit. Paidós.

Moscovici, S. (1986). El Psicoanálisis, su imagen y su público. Buenos Aires: Huemul.

Moscovicl. S., Hewstone, M. (1986). De la ciencia al sentido común. En: Moscovici, S. Psicología social II. Barcelona: Paidós.

$\mathrm{Ng}$, E., Ren, C. (2017). China's adaptation to climate and urban climatic changes: A critical review. Urban Climate, 23(1), 352-372 https:// doi.org/10.1016/j.uclim.2017.07.006

Niaz, M. (2016). History and Philosophy of Science as a Guide to Understanding Nature of Science. Revista Científica, 1(24), 7-16. https://doi. org/10.14483/udistrital.jour.RC.2016.24.a1

Pereira de Sá, C. (1998). A construçao do objeto de pesquisa en representaçoes sociais. Ed. Universidade Statal de Rio de Janeiro, Rio de Janeiro.

Porras Contreras, Y. A., Pérez Mesa, M. R. (2018). Identidad ambiental: múltiples perspectivas. Revista Científica, 1(34), 123-138. https://doi. org/10.14483/23448350.14003

Portillo Páez, F., Vega Vega, M., Cadavid Velásquez, E., Pérez Vásquez, N. (2017). Representaciones Sociales Sobre El Cambio Climático Y sus Efectos En Adolescentes de Instituciones Oficiales y Privadas De Montería - Córdoba. Bio-grafía, 10(19), 839-847. https://doi. org/10.17227/bio-grafia.extra2017-7217

Sánchez-Robayo, B. J., Torres-Duarte, J. (2016). Aprender a investigar investigando. Realización de una propuesta de formación. 
Revista Científica, 1(28), 17-31. https://doi. org/10.14483/udistrital.jour.RC.2017.28.a2

Vasilachis De Gialdino, I. (1992). Métodos cualitativos I: los problemas teórico-epistemológicos. Buenos Aires: Centro Editor de América Latina.
Wagner, W., Elejabarrieta, F. (1994). Representaciones sociales. En: J. Francisco Morales, M. Moya, E. Rebolloso, J. M. Fernández Dols, C. Huici, J. Marques, D. Páez y J. A. Pérez. Psicología social. McGraw-Hill, 